\title{
Perfil clínico, sociodemográfico e preditores de óbito em unidade de terapia intensiva
}

Clinical, sociodemographic profile and predictors of death in intensive care unit

Perfil clínico, sociodemográfico y predictores de muerte en la unidad de cuidados intensivos

\section{Josefine Busanello¹, Erica Gomes Quevedo ${ }^{\mathrm{II}}$, Ana Paula de Lima Escobal ${ }^{\mathrm{III}}$, Deisy Mello de Pinto $^{\mathrm{IV}}$, Natália Pinto Silveirav ${ }^{\mathrm{v}}$, Lucas Pitrez Mocellin ${ }^{\mathrm{VI}}$}

Resumo: Objetivo: identificar o perfil sociodemográfico e clínico dos pacientes de unidade de terapia intensiva do interior do Brasil e caracterizar os óbitos e os seus fatores preditores. Método: estudo transversal realizado a partir da análise documental de prontuários de amostra aleatória de pacientes em cuidados intensivos. Foram coletados dados entre $2016 \mathrm{e}$ 2018 utilizando instrumento padronizado. Análise descritiva realizada comparando óbitos e sobreviventes. Utilizou-se regressão multivariável para identificação dos preditores do óbito. Resultados: considerando os 259 pacientes analisados, ocorreram 231 mortes $(89,2 \%)$. Entre os óbitos, predominaram o sexo feminino $(55,4 \%)$, raça branca $(75,3 \%)$ e baixa escolaridade (66,7\%). As variáveis idade avançada, insuficiência cardíaca e infecção pelo vírus da imunodeficiência humana adquirida foram consideradas preditores do óbito. Conclusão: evidenciou-se a caracterização dos pacientes atendidos na terapia intensiva, assim como a identificação de sujeitos com maior risco de morte, fornecendo subsídios para a elaboração de estratégias efetivas de prevenção do óbito.

Descritores: Perfil de saúde; Mortalidade; Fatores de Risco; Unidades de Terapia Intensiva; Cuidados Críticos

Abstract: Objective: to identify the sociodemographic and clinical profile of patients in an intensive care unit in the interior of Brazil and to characterize the deaths and their predictors. Method: cross-sectional study conducted

\footnotetext{
${ }^{1}$ Enfermeira, Doutora em Enfermagem, Universidade Federal do Pampa, Uruguaiana, RS, Brasil. E-mail: josefinebusanello@unipampa.edu.br, ORCID: 0000-0002-9950-9514

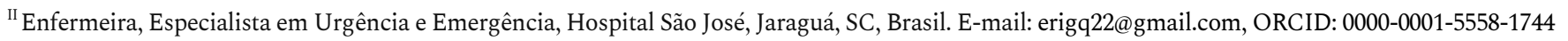

III Enfermeira, Doutora em Enfermagem, Universidade Federal do Pampa, Uruguaiana, RS, Brasil. E-mail: anapaulaescobal@hotmail.com, ORCID: 0000-0002-2601-9098

IV Enfermeira, Especialista em Urgência e Emergência, Hospital Santa Casa de Uruguaiana, RS, Brasil. E-mail: deisymellopinto@gmail.com, ORCID: 0000-0003-0818-8645

v Acadêmica do Curso de Enfermagem, Universidade Federal do Pampa, Uruguaiana, RS, Brasil. E-mail: nattaliapin@gmail.com, ORCID: 00000003-2322-2063

VI Professor do Ensino Superior, Doutor em epidemiologia, Universidade Federal do Pampa, Uruguaiana, RS, Brasil. E-mail: lucasmocellin@unipampa.edu.br, ORCID: 0000-0002-1766-9857
} 
from documentary analysis of medical records of a random sample of patients in intensive care. Data were collected between 2016 and 2018 using standardized instrument. Descriptive analysis performed comparing deaths and survivors. Multivariable regression was used to identify predictors of death. Results: considering the 259 patients analyzed, there were 231 deaths $(89.2 \%)$. Among the deaths, female gender (55.4\%), white race (75.3\%) and low education (66.7\%) predominated. The variables advanced age, heart failure, and human immunodeficiency virus infection were considered predictors of death. Conclusion: the characterization of patients seen in intensive care was evidenced, as well as the identification of subjects at higher risk of death, providing subsidies for the development of effective strategies to prevent death.

Descriptors: Health Profile; Mortality; Risk Factors; Intensive Care Units; Critical Care

Resumen: Objetivo: identificar el perfil sociodemográfico y clínico de los pacientes de una unidad de cuidados intensivos del interior de Brasil y caracterizar las muertes y sus factores predictores. Método: estudio transversal realizado a partir de un análisis documental de las historias clínicas de una muestra aleatoria de pacientes en cuidados intensivos. Los datos se recogieron entre 2016 y 2018 utilizando un instrumento estandarizado. Se realizó un análisis descriptivo comparando las muertes y los supervivientes. Se utilizó una regresión multivariable para identificar los predictores de muerte. Resultados: considerando los 259 pacientes analizados, se produjeron 231 muertes $(89,2 \%)$. Entre las muertes, predominaron el sexo femenino $(55,4 \%)$, la raza blanca $(75,3 \%)$ y la baja escolaridad $(66,7 \%)$. Las variaciones de la edad avanzada, la insuficiencia cardíaca y la infección por virus de la inmunodeficiencia humana adquirida se consideraron predictores de la muerte. Conclusión: se evidenció la caracterización de los pacientes atendidos en la terapia intensiva, así como la identificación de los sujetos con mayor riesgo de muerte, suministrando subsidios para la elaboración de estrategias efectivas de prevención de la muerte.

Descriptores: Perfil de Salud; Mortalidad; Factores de Riesgo; Unidades de Cuidados Intensivos; Cuidados Críticos

\section{Introdução}

A Unidade de Terapia Intensiva (UTI) é considerada um ambiente de alta complexidade hospitalar, destinada a pacientes em situações graves. Apesar dos avanços tecnológicos e assistenciais, as taxas de mortalidade dos pacientes ainda são elevadas. ${ }^{1-7}$ Estudo realizado no referido espaço hospitalar, no Japão, evidenciou taxa de mortalidade de $23 \% .{ }^{1}$ No Reino Unido, a mortalidade acomete $25,7 \%$ dos pacientes, ${ }^{2}$ enquanto, no Brasil, pesquisas demonstram uma taxa de mortalidade variando de 20,4 a $50 \%$. A gravidade das doenças e a idade avançada são indicadas como os principais preditores para o óbito..$^{3-7}$

A mortalidade dos pacientes também está associada às características clínicas e sociais, aos eventos adversos e às iatrogenias. A análise dos indicadores de qualidade assistencial, comumente utilizados na UTI, tais como o tempo de internação e as taxas de infecção, de igual 
3 | Busanello J, Quevedo EG, Escobal APL, Pinto DM, Silveira NP, Mocellin LP

forma, pode auxiliar na compreensão dos fatores associados à mortalidade dos pacientes de uma determinada unidade. ${ }^{1-8}$ A escassez de profissionais intensivistas também prejudica os resultados e o desfecho clínico para os pacientes graves. Em muitos contextos, esse problema é superado com investimentos em serviços de teleducação, objetivando capacitar e assessorar os profissionais atuantes em UTI alocada em cenário com menos recurso. ${ }^{9-10}$

As fragilidades do sistema de saúde influenciam nos índices de mortalidade dos pacientes, especialmente em regiões do interior, onde há menor desenvolvimento econômico, escassez de recursos na área da saúde, dispersão populacional e grandes distâncias geográficas. ${ }^{11}$ Essas situações condicionam o déficit de leitos e de condições estruturais para garantir o cuidado progressivo aos pacientes graves. ${ }^{12}$

Cabe ressaltar que há uma multiplicidade de situações clínicas e cirúrgicas que definem a característica generalista da maioria das UTIs. Isso, pois as particularidades da rede de atenção em saúde e a condição de saúde da população de localidades interioranas são determinantes para o perfil assistencial dessas unidades. ${ }^{11}$ Nesse contexto, a delimitação das características clínicas e epidemiológicas dos pacientes pode explicar o perfil da mortalidade, especialmente as taxas e os fatores de risco para os óbitos. Essa perspectiva pode auxiliar na identificação das principais fragilidades assistenciais e na promoção de ações de assessoramento e qualificação das UTIs, contribuindo para a redução dos riscos de complicações e da mortalidade.

Nesse sentido, questiona-se: qual o perfil sociodemográfico e clínico dos pacientes de unidade de terapia intensiva do interior do Brasil? Quais são os fatores preditores dos óbitos? O presente estudo objetivou identificar o perfil sociodemográfico e clínico dos pacientes de unidade de terapia intensiva do interior do Brasil e caracterizar os óbitos e os seus fatores preditores. 
Perfil clínico, sociodemográfico e preditores de óbito em unidade de terapia intensiva $\mid 4$

\section{Método}

Estudo transversal realizado a partir da análise documental de prontuários de pacientes internados em uma unidade de terapia intensiva adulto, entre os anos de 2016 a 2018, de um hospital geral do interior do Brasil, situado em um município com uma população estimada de 127 mil pessoas. A instituição hospitalar possui caráter público/privado, estando vinculada ao Sistema Único de Saúde, e presta assistência à saúde em áreas básicas e em especialidades, com média e alta complexidade, condicionando-se como referência para a população locoregional. A UTI dispõe de dez leitos, sendo referência em neurologia e neurocirurgia.

Foram considerados como critérios de inclusão: prontuários de pacientes atendidos na UTI adulto (idade igual ou superior a 18 anos) no período de 2016 a 2018. Foram excluídos os prontuários de pacientes que permaneceram menos de 24 horas de internação na unidade. Tal critério foi adotado devido a não completude dos registros frente à inviabilidade de tempo para o histórico de informações essenciais que se configuram como variáveis do estudo.

O cálculo amostral baseou-se no total de pacientes que estiveram internados na UTI e na estimativa da taxa de mortalidade entre os sujeitos que acessaram esse serviço. Foi utilizada uma margem de erro de 5\%, tamanho da população estudada de 419 indivíduos (140 a cada ano) e estimativa da taxa de mortalidade de 40\%. Um total de 195 indivíduos seriam necessários para inclusão no estudo. Realizou-se a numeração dos prontuários de pacientes que passaram pela UTI no período do estudo e, sequencialmente, o processo de seleção foi estabelecido a partir de números aleatórios gerados eletronicamente por meio do software Statistical Package for Social Sciences $^{\circledR}$ (SPSS), versão 22.0. Caso o indivíduo selecionado não possuísse os critérios de inclusão referidos, o número subsequente que atendesse os critérios para participação era selecionado.

Para a coleta das informações, foi elaborado um instrumento a partir da análise prévia dos prontuários dos pacientes admitidos na UTI, e a seleção das variáveis sociodemográficas e 
5 | Busanello J, Quevedo EG, Escobal APL, Pinto DM, Silveira NP, Mocellin LP

clínicas, para compor esse instrumento, foi fundamentada na literatura científica. ${ }^{1-7}$ Foram consideradas as seguintes variáveis: idade; sexo; profissão; cor/raça; município de origem; estado civil; escolaridade; religião; diagnóstico no momento da internação; comorbidades; tempo total em dias completos de internação na UTI; uso da ventilação mecânica e sedação. Também foi verificado o desfecho clínico dos pacientes, sobrevida ou óbito, sendo o último considerado a variável de desfecho no estudo.

Previamente à realização da coleta de dados, averiguou-se a pertinência das variáveis do instrumento por meio da consulta a especialistas com experiência em assistência na UTI. A busca por informações foi realizada no período de agosto a setembro de 2019, no Serviço de Arquivo Médico e Estatística (SAME) do cenário do estudo. O acesso às informações dos pacientes ocorreu por meio de prontuários em papel e eletrônicos. Estes estavam organizados por mês e ano em que aconteceu a internação hospitalar dos pacientes. Os pesquisadores envolvidos nessa etapa foram previamente treinados para a execução da atividade, de forma a padronizá-la e evitar viés de informação no estudo.

Os dados coletados foram organizados em um arquivo no gerenciador de planilhas Microsoft Excel versão 16.0. Para o tratamento quantitativo, utilizou-se o software SPSS e, para as análises descritivas, utilizou-se a distribuição de frequências, as quais foram desenvolvidas comparando indivíduos que foram a óbito com os que sobreviveram. Comparações entre as especialidades médicas na UTI e suas respectivas taxas de mortalidade foram desenvolvidas. O indicador referido foi calculado utilizando como numerador os óbitos e denominador o número de pacientes atendidos multiplicado pelo fator cem (óbitos/100 casos atendidos). Por fim, realizou-se uma análise multivariável, a fim de identificar fatores preditores do óbito na população estudada. Variáveis que foram consideradas associadas à morte, segundo evidências científicas, $^{1-8}$ como a idade, escolaridade, tempo de internação, presença de comorbidades (diabetes, hipertensão, insuficiência cardíaca e infecção pelo vírus da imunodeficiência humana 
Perfil clínico, sociodemográfico e preditores de óbito em unidade de terapia intensiva |6

- HIV), uso de ventilação mecânica e o uso de sedação foram incluídas na análise multivariável, realizando-se, dessa forma, o ajuste dos referidos fatores para a identificação de fatores preditores do óbito, minimizando-se o viés de confusão no estudo. Utilizou-se o método de regressão de Poisson com variância robusta, o qual estima como medida de associação a razão de prevalência. O nível de significância $(\alpha)$ foi definido em 0,05 .

O presente estudo foi aprovado pelo Comitê de Ética e Pesquisa, sob o número do parecer 3.404.096, com CAAE: 12237519.4.0000.5323, no dia 20 de junho de 2019. A pesquisa dispensou o uso do Termo de Consentimento Livre e Esclarecido. Respeitaram-se as perspectivas legais e éticas, especialmente, a confidencialidade e a privacidade dos dados coletados, conforme preconiza a resolução 466/2012 do Conselho Nacional de Saúde.

\section{Resultados}

Um total de 419 pacientes foram internados no período de 2016 a 2018. A partir da amostragem aleatória, foram selecionados 259 prontuários, representando $61,8 \%$ de todos os que estiveram na UTI. Um total de 231 óbitos ocorreram $(89,2 \%)$ na amostra estudada. Na Tabela 1 , estão apresentadas as características sociodemográficas dos participantes da pesquisa, comparando os óbitos com os sobreviventes.

Tabela 1 - Características sociodemográficas dos pacientes internados em UTI e que foram a óbito e dos sobreviventes na instituição participante do estudo, de 2016 a 2018 (n=259).

\begin{tabular}{|c|c|c|c|c|}
\hline \multirow{2}{*}{ Variáveis } & \multicolumn{2}{|c|}{ Óbitos (n=231) } & \multicolumn{2}{|c|}{ Sobreviventes $(\mathrm{n}=28)$} \\
\hline & $\mathbf{n}$ & $\%$ & $\mathbf{n}$ & $\%$ \\
\hline Sexo Feminino & 128 & 55,4 & 14 & 50,0 \\
\hline \multicolumn{5}{|l|}{ Faixa etária } \\
\hline Até 51 anos & 58 & 25,1 & 9 & 32,1 \\
\hline 51 a 64 anos & 59 & 25,5 & 7 & 25,0 \\
\hline 64 a 73 anos & 55 & 23,8 & 8 & 28,6 \\
\hline Mais de 73 anos & 59 & 25,5 & 4 & 14,3 \\
\hline \multicolumn{5}{|l|}{ Escolaridade } \\
\hline Até ensino fundamental completo & 154 & 66,7 & 22 & 78,6 \\
\hline
\end{tabular}




\begin{tabular}{|c|c|c|c|c|}
\hline Acima de ensino fundamental completo & 57 & 24,7 & 5 & 17,9 \\
\hline Ignorado & 20 & 8,7 & 1 & 3,6 \\
\hline \multicolumn{5}{|l|}{ Raça/cor } \\
\hline Branco & 174 & 75,3 & 16 & 57,1 \\
\hline Pardo/Negro & 9 & 3,9 & 2 & 7,1 \\
\hline Ignorado & 48 & 20,8 & 10 & 35,7 \\
\hline \multicolumn{5}{|l|}{ Situação de trabalho } \\
\hline Aposentado & 83 & 35,9 & 7 & 25,0 \\
\hline Trabalhando & 57 & 24,7 & 9 & 32,1 \\
\hline Desempregado/do lar & 43 & 18,6 & 5 & 17,9 \\
\hline Ignorado & 48 & 20,7 & 7 & 25,0 \\
\hline \multicolumn{5}{|l|}{ Religião } \\
\hline Católica & 129 & 55,8 & 17 & 60,7 \\
\hline Outras & 49 & 21,2 & 3 & 10,7 \\
\hline Ignorado & 53 & 22,9 & 8 & 28,6 \\
\hline \multicolumn{5}{|l|}{ Estado civil } \\
\hline Casados ou união estável & 84 & 36,4 & 11 & 39,3 \\
\hline Solteiro/divorciado/viúvo & 131 & 56,7 & 16 & 57,1 \\
\hline Ignorado & 16 & 6,9 & 1 & 3,6 \\
\hline \multicolumn{5}{|l|}{ Origem } \\
\hline Pertence ao município sede da instituição hospitalar & 163 & 70,6 & 11 & 39,3 \\
\hline Não pertence ao município sede da instituição hospitalar & 68 & 29,4 & 17 & 60,7 \\
\hline
\end{tabular}

Predominou pacientes do sexo feminino (55,4\%) entre os óbitos, enquanto foi observado o mesmo número de homens e mulheres entre os sobreviventes. Prevaleceu a faixa etária com até 51 anos $(32,1 \%)$ entre os sobreviventes e as categorias de 51 a 64 anos e mais de 73 anos entre os indivíduos que faleceram (25,5\% para ambas), embora a distribuição tenha se apresentado bem equilibrada entre as quatro faixas etárias.

Evidenciou-se a baixa escolaridade dos pacientes, sendo a maioria restrita ao ensino fundamental completo, considerando os óbitos $(66,7 \%)$ e os sobreviventes $(78,6 \%)$. Também se observou maior frequência da raça branca $(75,3 \%$ e $57,1 \%)$ e dos solteiros, divorciados ou viúvos (56,7\% e 57,1\%), respectivamente para os óbitos e sobreviventes. Para a variável situação de trabalho, verificou-se que entre os falecidos a maior frequência foi de aposentados $(35,9 \%)$, enquanto para os indivíduos que se recuperaram foram de trabalhadores vigentes $(32,1 \%)$. 
Perfil clínico, sociodemográfico e preditores de óbito em unidade de terapia intensiva $\mid 8$

Em relação à variável origem do paciente, observa-se que 70,6\% dos óbitos foram de indivíduos pertencentes ao município sede da instituição hospitalar, ao passo que $60,7 \%$ dos sobreviventes não pertencem ao município.

Em relação às características clínicas, destaca-se o predomínio da especialidade de neurologia entre óbitos $(31,2 \%)$ e sobreviventes $(42,9 \%)$ (Tabela 2). Foi verificada a presença de comorbidades, especialmente, hipertensão arterial sistêmica (40,3\% e 39,3\%), diabetes mellitus $(24,2 \%$ e $32,1 \%)$, acidente vascular encefálico $(15,2 \%$ e $14,3 \%)$ e doença pulmonar obstrutiva crônica (DPOC) $(15,2 \%$ e $10,7 \%)$, respectivamente para pacientes que morreram e os que se recuperaram. A maioria dos pacientes foi submetido à ventilação mecânica entre óbitos $(77,9 \%)$ e sobreviventes $(89,3 \%)$. O tempo de internação apresentou predomínio da categoria até dois dias $(32,9 \%)$ entre os óbitos, ao passo que os sobreviventes demonstraram mais de nove dias $(35,7 \%)$.

Tabela 2 - Características clínicas dos pacientes internados em UTI que foram a óbito e dos sobreviventes na instituição participante do estudo, RS, Brasil, de 2016 a 2018 ( $\mathrm{n}=259)$.

\begin{tabular}{|c|c|c|c|c|}
\hline \multirow{2}{*}{ Variáveis } & \multicolumn{2}{|c|}{ Óbitos (n=231) } & \multicolumn{2}{|c|}{ Sobreviventes $(\mathrm{n}=28)$} \\
\hline & $\mathrm{n}$ & $\%$ & $\mathbf{n}$ & $\%$ \\
\hline \multicolumn{5}{|l|}{ Especialidade } \\
\hline Neurologia & 72 & 31,2 & 12 & 42,9 \\
\hline Pneumologia & 66 & 28,6 & 5 & 17,9 \\
\hline Cardiologia & 28 & 12,1 & 5 & 17,9 \\
\hline Nefrologia & 18 & 7,8 & 1 & 3,6 \\
\hline Gastroenterologia & 15 & 6,5 & 1 & 3,6 \\
\hline Oncologia & 7 & 3,0 & 1 & 3,6 \\
\hline Obstetrícia & 2 & 0,9 & 3 & 10,7 \\
\hline Outras & 23 & 9,9 & - & - \\
\hline \multicolumn{5}{|l|}{ Pacientes com agravos de saúde } \\
\hline Hipertensão Arterial Sistêmica & 93 & 40,3 & 11 & 39,3 \\
\hline Diabetes Mellitus & 56 & 24,2 & 9 & 32,1 \\
\hline Insuficiência Cardíaca Congestiva & 22 & 9,5 & - & - \\
\hline Insuficiência Renal Crônica & 17 & 7,4 & 2 & 7,1 \\
\hline Acidente Vascular Encefálico & 35 & 15,2 & 4 & 14,3 \\
\hline DPOC & 35 & 15,2 & 3 & 10,7 \\
\hline Enfisema pulmonar & 3 & 1,3 & - & - \\
\hline Tuberculose & 6 & 2,6 & - & - \\
\hline
\end{tabular}


9 | Busanello J, Quevedo EG, Escobal APL, Pinto DM, Silveira NP, Mocellin LP

\begin{tabular}{lcccc} 
Pneumonia & 10 & 4,3 & 1 & 3,6 \\
Câncer & 14 & 6,1 & 1 & 3,6 \\
HIV & 13 & 5,6 & - & - \\
Sífilis & 1 & 0,4 & - & - \\
$\quad$ Hepatites & 1 & 0,4 & 1 & 3,6 \\
Tempo de internação & & & & \\
Até 2 dias & 76 & 32,9 & 7 & 25,0 \\
2 a 4 dias & 50 & 21,6 & 7 & 25,0 \\
4 a 9 dias & 55 & 23,8 & 4 & 35,3 \\
Mais de 9 dias & 50 & 21,6 & 10 & 89,3 \\
Ventilação Mecânica & 180 & 77,9 & 25 & 60,7 \\
Sedação & 115 & 49,8 & 17 & \\
\hline
\end{tabular}

DPOC: Doença Pulmonar Obstrutiva Crônica; HIV: vírus da imunodeficiência humana.

$\mathrm{Na}$ Tabela 3, apresentam-se os indicadores de mortalidade, considerando a causa da internação por especialidade. Observa-se que as maiores taxas de mortalidade (óbitos por 100 casos atendidos) estão nas especialidades de nefrologia $(94,74)$, gastroenterologia $(93,75)$ e pneumologia (92,96). A referida taxa em neurologia também é alta $(85,71)$ e a menor foi da especialidade de obstetrícia $(40,0)$.

Tabela 3 - Indicadores de mortalidade por especialidade médica entre as internações em UTI na instituição participante do estudo, RS, Brasil, de 2016 a 2018 (n=236).

\begin{tabular}{lccc}
\hline Especialidade & Número de pessoas atendidas & Percentual & Taxa de mortalidade $^{*}$ \\
\hline Nefrologia & 19 & 8,05 & 94,74 \\
Gastroenterologia & 16 & 6,77 & 93,75 \\
Pneumologia & 71 & 30,08 & 92,96 \\
Oncologia & 8 & 3,38 & 87,50 \\
Neurologia & 84 & 35,59 & 85,71 \\
Cardiologia & 33 & 13,98 & 84,85 \\
Obstetrícia & 5 & 2,11 & 40,00 \\
\hline
\end{tabular}

*Óbitos por 100 casos atendidos

Conforme Tabela 4, foi realizada uma análise multivariada, a fim de identificar fatores preditores para o óbito em UTI. Percebe-se, primeiramente no modelo univariado, que a presença de insuficiência cardíaca $(\mathrm{p}<0,001 ; \mathrm{RP}=0,134)$ e do HIV ( $<<0,001 ; \mathrm{RP}=1,128)$ foram 
estatisticamente significativos, demonstrando risco para o óbito. A condição de residir no município sede da instituição hospitalar de $(\mathrm{p}=0,006)$ também demonstrou significância estatística, apresentando-se como fator de risco para o óbito ( $\mathrm{RP}=1,171)$.

Tabela 4 - Análise multivariada para identificação dos fatores preditores para o óbito entre pacientes atendidos na UTI da instituição participante do estudo, RS, Brasil, de 2016 a 2018 (n=259).

\begin{tabular}{|c|c|c|c|c|c|c|c|c|c|c|}
\hline \multirow{3}{*}{$\begin{array}{l}\text { Fatores } \\
\text { Idade } \geq 73 \text { anos }\end{array}$} & \multicolumn{5}{|c|}{ Univariado } & \multicolumn{3}{|c|}{ Multivariado $-\mathrm{N}=238$} & \multirow{2}{*}{\multicolumn{2}{|c|}{$\begin{array}{c}\mathrm{RV}=-234,879 \\
\mathrm{IC}(95 \%)\end{array}$}} \\
\hline & \multirow{2}{*}{$\begin{array}{l}n \\
63\end{array}$} & \multirow{2}{*}{$\begin{array}{c}\% \\
24,3\end{array}$} & \multirow{2}{*}{$\begin{array}{c}\mathrm{RP} \\
1,082\end{array}$} & \multicolumn{2}{|c|}{ IC (95\%) } & \multirow{2}{*}{$\begin{array}{c}\mathbf{n} \\
61\end{array}$} & \multirow{2}{*}{$\begin{array}{c}\% \\
25,6\end{array}$} & \multirow{2}{*}{$\begin{array}{c}\mathbf{R P} \\
1,158\end{array}$} & & \\
\hline & & & & 0,965 & 1,213 & & & & 1,001 & 1,340 \\
\hline Idade 64 a 73 anos & 63 & 24,3 & 1,008 & 0,883 & 1,152 & 58 & 24,4 & 1,086 & 0,921 & 1,281 \\
\hline Idade 51 a 64 anos & 66 & 25,5 & 1,033 & 0,911 & 1,171 & 61 & 25,6 & 1,089 & 0,935 & 1,267 \\
\hline Idade $\leq 51$ anos & 67 & 25,9 & 1,0 & - & - & 58 & 24,4 & 1,0 & - & - \\
\hline $\begin{array}{l}\text { Residente da cidade sede da } \\
\text { instituição Hospitalar }\end{array}$ & 174 & 67,2 & 1,171 & 1,046 & 1,311 & 159 & 66,8 & 1,173 & 1,043 & 1,319 \\
\hline Residente de outros municípios & 85 & 32,8 & 1,0 & - & - & 79 & 33,2 & 1,0 & - & - \\
\hline $\begin{array}{l}\text { Escolaridade ensino fundamental } \\
\text { completo }\end{array}$ & 176 & 73,9 & 1,051 & 0,958 & 1,152 & 176 & 73,9 & 1,041 & 0,937 & 1,155 \\
\hline Escolaridade ensino Médio ou mais & 62 & 26,1 & 1,0 & - & - & 62 & 26,1 & 1,0 & - & - \\
\hline Presença de DM & 65 & 25,1 & 0,955 & 0,857 & 1,064 & 62 & 26,1 & 0,940 & 0,846 & 1,045 \\
\hline Ausência de DM & 194 & 74,9 & 1,0 & - & - & 176 & 73,9 & 1,0 & - & - \\
\hline Presença de ICC & 22 & 8,5 & 1,134 & 1,082 & 1,188 & 21 & 8,8 & 1,174 & 1,068 & 1,292 \\
\hline Ausência de ICC & 237 & 91,5 & 1,0 & - & - & 217 & 91,2 & 1,0 & - & - \\
\hline Presença de HIV & 13 & 5,0 & 1,128 & 1,079 & 1,180 & 10 & 4,2 & 1,217 & 1,069 & 1,384 \\
\hline Ausência de HIV & 246 & 95,0 & 1,0 & - & - & 228 & 95,8 & 1,0 & - & - \\
\hline Presença de HAS & 104 & 40,2 & 1,004 & 0,921 & 1,095 & - & - & - & - & - \\
\hline Ausência de HAS & 155 & 59,8 & 1,0 & - & - & - & - & - & - & - \\
\hline 9 dias ou mais de internação & 60 & 23,2 & 0,910 & 0,799 & 1,037 & 59 & 24,8 & 0,931 & 0,820 & 1,056 \\
\hline De 4 a 9 dias de internação & 59 & 22,8 & 1,018 & 0,926 & 1,119 & 50 & 21,0 & 1,062 & 0,963 & 1,171 \\
\hline De 2 a 4 dias de internação & 57 & 22,0 & 0,958 & 0,852 & 1,077 & 53 & 22,3 & 0,987 & 0,869 & 1,119 \\
\hline Até 1 dia de internação & 83 & 32,0 & 1,0 & - & - & 76 & 31,9 & 1,0 & - & - \\
\hline
\end{tabular}


11 | Busanello J, Quevedo EG, Escobal APL, Pinto DM, Silveira NP, Mocellin LP

\begin{tabular}{lccccccccccc} 
Especialidade outros & 71 & 27,4 & 1,068 & 0,955 & 1,195 & 66 & 27,7 & 1,012 & 0,909 & 1,127 \\
Especialidade respiratório & 71 & 27,4 & 1,085 & 0,973 & 1,209 & 64 & 26,9 & 1,029 & 0,924 & 1,146 \\
Especialidade cardiológico & 33 & 12,7 & 0,990 & 0,836 & 1,172 & 31 & 13,0 & 0,922 & 0,722 & 1,102 \\
Especialidade neurológico & 84 & 32,4 & 1,0 & - & - & 77 & 32,4 & 1,0 & - & - \\
Uso de VM & 205 & 79,2 & 0,930 & 0,856 & 1,010 & - & - & - & - & - \\
Não usou VM & 54 & 20,8 & 1,0 & - & - & - & - & - & - & - \\
Uso Sedação & 132 & 51,0 & 0,954 & 0,876 & 1,038 & - & - & - & - & - \\
Não usou Sedação & 127 & 49,0 & 1,0 & - & - & - & - & - & - & - \\
\hline
\end{tabular}

RV: Razão de Verossimilhança; RP: Razão de Prevalência; IC: Intervalo de Confiança; DM: Diabetes Mellitus; HAS: hipertensão arterial sistêmica; ICC: Insuficiência Cardíaca Congestiva; VM: Ventilação Mecânica.

No modelo multivariável, em que se verifica a influência dos fatores preditores para o óbito ajustando para todas as variáveis incluídas no modelo, foram selecionados: idade, município de origem, escolaridade, diabetes, insuficiência cardíaca, infecção pelo HIV, tempo de internação e especialidade no atendimento. As variáveis que apresentaram significância estatística foram: idade, para a maior faixa etária (maior que 73 anos) (valor $\mathrm{p}=0,050$; RP = 1,158); pertencer ao município sede da instituição hospitalar ( $\mathrm{p}=0,008 ; \mathrm{RP}=1,173)$; presença de insuficiência cardíaca $(\mathrm{p}=0,001 ; \mathrm{RP}=1,174)$ e de infecção pelo vírus $\mathrm{HIV}(\mathrm{p}=0,003 ; \mathrm{RP}=1,217)$. Todos os fatores listados apresentaram associação indicando risco para o óbito. Não houve significância estatística para as demais variáveis incluídas no modelo.

\section{Discussão}

Os achados do perfil social do paciente internado em uma UTI interiorana no Brasil corroboram, parcialmente, com as publicações científicas para esse enfoque. Foi constatado que houve predominância de pacientes do sexo feminino, diferentemente de outros estudos que evidenciaram o predomínio do sexo masculino. ${ }^{2-5} \mathrm{~A}$ maioria dos pacientes possui baixa escolaridade, fato que vai ao encontro de pesquisa realizada no Nordeste brasileiro, que 
Perfil clínico, sociodemográfico e preditores de óbito em unidade de terapia intensiva | 12

evidenciou que $63,5 \%$ dos pacientes internados na UTI possuem apenas ensino fundamental completo. ${ }^{13}$ A média de idade dos pacientes é de 61 anos, resultado semelhante ao apontado na literatura. ${ }^{2-5} \mathrm{O}$ envelhecimento populacional tende a aumentar a demanda dos serviços de atendimento intensivo e está diretamente relacionada ao aumento do óbito. ${ }^{7}$ Além disso, a idade foi considerada um fator preditor do óbito, apresentando significância estatística na análise multivariada (categoria maior ou igual a 73 anos).

O presente estudo demonstrou variabilidade das especialidades atendidas, refletindo a característica generalista das UTIs interioranas do Brasil. A especialidade de neurologia superou, levemente, as demais especialidades (cardiologia, pneumologia e outras). As doenças cerebrovasculares são uma das principais causas de morbidade e mortalidade. Esse mesmo achado foi evidenciado em pesquisas que destacam que o paciente neurocrítico requer suporte e tecnologia para garantir a assistência de alta complexidade., ${ }^{3,5} \mathrm{Em}$ outra publicação, foi constatada a prevalência de acidente vascular cerebral e insultos neurológicos não traumáticos, como situações que predominam no referido ambiente hospitalar. ${ }^{7}$ Apesar da especialidade de neurologia ser prevalente, observou-se que a taxa de mortalidade mais elevada ocorreu na especialidade de nefrologia (94,7 óbitos a cada 100 casos), seguido pelas especialidades gastroenterologia e pneumologia. A letalidade envolvendo pacientes com comprometimento renal pode estar associada à instabilidade hemodinâmica que prevalece nesse fenótipo e às complicações associadas à terapia de substituição renal.

A hipertensão arterial sistêmica é apontada como o relevante fator de risco para o desenvolvimento de eventos neurológicos graves, ${ }^{3}$ o que vai ao encontro dos achados desta pesquisa, uma vez que a referida enfermidade foi a mais frequente entre as comorbidades, apesar de não ter se mostrado estatisticamente significativo na análise multivariada. Outra comorbidade que teve relevância foi o diabetes mellitus, apresentando-se como o segundo evento clínico mais frequente. Na literatura, ela é ressaltada como uma das condições crônicas 
13 | Busanello J, Quevedo EG, Escobal APL, Pinto DM, Silveira NP, Mocellin LP

mais prevalentes em pacientes críticos. ${ }^{3}$ A presença de insuficiência cardíaca congestiva ou infecção pelo HIV foram evidenciados como fator de risco para o óbito do paciente internado na UTI. Em outras pesquisas, a insuficiência cardíaca foi constatada como causa de óbito nos pacientes graves. ${ }^{3,7}$ As comorbidades são importantes preditores de morte a curto prazo e sua existência minimiza a possibilidade de se obter melhora do prognóstico. ${ }^{1}$ Nesse sentido, destaca-se a importância da avaliação clínica e da identificação das comorbidades no momento da internação do paciente na UTI, com o intuito de considerá-las no plano terapêutico e evitar o desencadeamento de complicações associadas.

O uso da ventilação mecânica e da sedação são medidas assistenciais necessárias para o manejo da maioria das situações críticas de vida. Especificamente, a ventilação mecânica invasiva visa a manutenção da oxigenação e ventilação dos pacientes com insuficiência respiratória aguda ou crônicas agudizadas. A sedação também está relacionada ao uso da ventilação mecânica invasiva, visto que é requisito inicial para estabelecer o suporte ventilatório avançado. ${ }^{14}$ Pesquisas destacam que a utilização da sedação pode desencadear delirium e prolongar o tempo de utilização da ventilação mecânica, aumentando o risco de mortalidade em $10 \%$ ao dia., ${ }^{214}$ Situação diferente da do presente estudo, em que o uso de ventilação mecânica e/ou sedação não foram evidenciados como foram fatores preditores para o óbito.

Os dados de tempo de internação demonstraram maior período entre sobreviventes, em comparação aos óbitos, assemelhando-se aos achados de outras pesquisas. ${ }^{4,7}$ Por outro lado, o tempo de internação na UTI não apresentou associação estatisticamente significativa com a mortalidade na presente pesquisa. Esse fato pode estar associado à condição grave e às disfunções orgânicas irreversíveis apresentadas pelo paciente no momento da internação, que, geralmente, é retardada pela espera de leito/vaga em terapia intensiva, contribuindo para o óbito nos primeiros dias de UTI. Em um estudo que comparou a taxa de óbito entre instituições públicas e privadas, essa relação teve significância estatística. ${ }^{4}$ 
Perfil clínico, sociodemográfico e preditores de óbito em unidade de terapia intensiva | 14

Nas unidades públicas, o tempo total de internação é, em média, de 10,59 dias, e a mortalidade chega a 25,52\%. Nas UTIs privadas, a média é de sete dias de internação, e a mortalidade atinge apenas $14,52 \%$ dos pacientes.

O estudo demonstrou que quase $90 \%$ dos pacientes atendidos na UTI foram a óbito, evidenciando uma taxa de mortalidade quase duas vezes maior quando comparada com outras publicações. ${ }^{1-6}$ Esse resultado também pode representar a realidade de outras instituições hospitalares interioranas do Brasil, obscurecidas pela escassez de pesquisas em UTIs que enfrentam as mesmas fragilidades do sistema de saúde. ${ }^{13}$ Ainda, a maioria dos artigos científicos que analisam o perfil de saúde do paciente da UTI é desenvolvida em instituições de referência de alta complexidade e centros de excelência em cuidados críticos..$^{1-3,5-7,14-15}$ Nessas instituições é esperada uma taxa de óbito inferior, pois a estrutura da rede de atenção, incluindo das instituições hospitalares e UTIs, garantem, minimamente, o suporte necessário para o cuidado progressivo ao paciente crítico. ${ }^{12}$

Uma pesquisa evidenciou que o risco de óbito nos hospitais de pequeno e médio porte é quase duas vezes o risco de óbito nos hospitais de grande porte. ${ }^{16}$ Ademais, taxas de mortalidade hospitalar são mais altas em cenários em que o recurso é limitado. ${ }^{10}$ Situação que ficou evidente a partir da pandemia associada ao Severe Acute Respiratory Syndrome Coronavirus 2 (SARS-COv-2), em que os sistemas de saúde enfrentam sérias restrições na capacidade e acessibilidade aos serviços de diagnóstico e de terapia intensiva, desencadeando piores resultados clínicos e maior mortalidade. ${ }^{17}$

No Brasil, essa situação se apresenta de forma mais complexa nas regiões interioranas, onde há o maior déficit de leitos de UTI. ${ }^{12,18-19}$ Levantamento realizado pela Associação de Medicina Intensiva Brasileira em 2016 mostrou que a proporção de leitos de UTI para cada 10 mil habitantes no país foi de $4,26 \%$ para as capitais e de apenas $1,33 \%$ para o interior. ${ }^{12} \mathrm{~A}$ discrepância é ainda maior na região Nordeste e Norte do Brasil $(4,02 \%$ e $0,60 \%$, 
15 | Busanello J, Quevedo EG, Escobal APL, Pinto DM, Silveira NP, Mocellin LP

respectivamente). No Rio Grande do Sul, verifica-se na capital, Porto Alegre, uma taxa de 6,30\% de leitos de UTI para cada 10 mil habitantes, enquanto que no interior do estado o valor é de 1,47\% para essa mesma taxa. ${ }^{12}$ Conforme pesquisa realizada no estado do Rio de Janeiro, necessita-se de um incremento de $122,0 \%$ de leitos para garantir a estabilidade do sistema e de 134,0\% para um tempo máximo de espera de seis horas. ${ }^{20}$ Estudos também comprovaram que, quanto maior tempo de espera do paciente grave por leito de UTI, maior a mortalidade nesse grupo. ${ }^{15,19}$

Além de aumentar o número de leitos, outras ações de gestão podem contribuir para a redução do índice de óbitos na UTI: fortalecimento das centrais de regulação de leitos; desenvolvimento de protocolos que priorizam o atendimento dos pacientes mais graves; ampliação dos leitos de retaguarda que permitam a alta do paciente da UTI, quando em condições clínicas; qualificação do atendimento pré-hospitalar e emergências; e educação permanente para a equipe multiprofissional. ${ }^{18-19}$

Cabe ainda destacar que, no que diz respeito às variáveis associadas ao óbito, pertencer ao município sede da instituição hospitalar pesquisada foi considerado fator de risco para a morte. Informações sobre a rede de serviços intermunicipais da região merecem maior detalhamento para a compreensão desse achado. Pequenas cidades encaminham os pacientes para a UTI do estudo, que é referência em alta complexidade de neurologia na região. A concessão de leitos tem como principal critério o diagnóstico/hipótese diagnóstica e o transporte/transferência intermunicipal do paciente que ocorre mediante sua estabilização clínica. Receber essa assistência prévia à internação na UTI, ainda no município de origem, pode contribuir para o desfecho com melhores resultados desses pacientes.

Uma das limitações da presente pesquisa é a não utilização de escalas para predispor o risco de morte dos pacientes. Outra questão a ser considerada é a utilização de dados secundários na presente pesquisa, que depende da qualidade e disponibilidade da informação. 
Perfil clínico, sociodemográfico e preditores de óbito em unidade de terapia intensiva | 16

Cabe ressaltar também as dificuldades acerca da completitude dos registros dos prontuários, o que torna o trabalho da equipe de pesquisa ainda mais desafiador.

\section{Conclusão}

Entre os achados, considerando o total de 259 pacientes analisados, ocorreram 231 mortes $(89,2 \%)$. Entre os óbitos, predominaram o sexo feminino $(55,4 \%)$, raça branca $(75,3 \%)$ e baixa escolaridade (66,7\%). As variáveis idade, insuficiência cardíaca e infecção pelo HIV foram considerados preditores do óbito.

Destaca-se a importância de desenvolver estudos multicêntricos que incluam na pesquisa UTIs interioranas do Brasil para conhecer outras realidades. Também ficou evidente a necessidade de contextualizar os fatores preditores para o óbito frente à organização e aos recursos da rede de atenção em saúde.

Os resultados do estudo permitiram a identificação de sujeitos com maior risco de morte, possibilitando a elaboração de estratégias efetivas de prevenção do óbito no ambiente do cuidado intensivo ao paciente. Além disso, contribui na fundamentação e replanejamento das ações de saúde no âmbito da UTI, assim como na revisão e atualização dos protocolos assistenciais. Ainda, os achados ressaltam a relevância de realizar-se a articulação com a rede de atenção local para a regulação de leitos, colaborando para o planejamento estratégico e organizacional dos serviços de saúde.

\section{Referências}

1. Abe T, Ogura H, Shiraishi A, Kushimoto S, Saitoh D, Fujishima S, et al. Characteristics, management, and in-hospital mortality among patients with severe sepsis in intensive care units in Japan: the FORECAST study. Crit Care. 2018 Nov 22;22:322. doi: 10.1186/s13054-018-2186-7

2. Gershengorn HB, Harrison DA, Garland A, Wilcox ME, Rowan KM, Wunsch H. Association of Intensive Care Unit Patient-to-Intensivist ratios with hospital mortality. JAMA Intern Med. 2017 
17 | Busanello J, Quevedo EG, Escobal APL, Pinto DM, Silveira NP, Mocellin LP

Mar;177(3):388-96. doi: 10.1001/jamainternmed.2016.8457

3. Vieira AM, Parente EA, Oliveira LS, Queiroz AL, Bezerra ISAM, Rocha HAL. Características de óbitos dos pacientes internados em uma unidade de terapia intensiva de hospital terciário. J Health Biol Sci. 2019;7(1):26-31. doi: 10.12662/2317-3076jhbs.v7i1.1999.p26-31.2019

4. Nogueira LS, Sousa RMC, Padilha KG, Koike KM. Clinical characteristics and severity of patients admitted to public and private ICUs. Texto Contexto Enferm. 2012;21(1):59-67. doi: 10.1590/S010407072012000100007

5. Rodriguez AH, Bub MBC, Perão OF, Zandonadi G, Rodriguez MJH. Epidemiological characteristics and causes of deaths in hospitalized patients under intensive care. Rev Bras Enferm. 2016;69(2):210-4. doi: 10.1590/0034-7167.2016690204i

6. Lentsck MH, Oliveira RR, Corona LP, Mathias TAF. Risk factors for death of trauma patients admitted to an Intensive Care Unit. Rev Latinoam Enferm. 2020 Feb 14;28:e3236. doi: 10.1590/15188345.3482 .3236

7. Gulini JEHMB, Nascimento ERP, Moritz RD, Vargas MAO, Matte DL, Cabral RP. Predictors of death in an Intensive Care Unit: contribution to the palliative approach. Rev Esc Enferm USP. 2018 Jun 25;52:e03342. doi: 10.1590/S1980-220X2017023203342

8. Cordeiro P, Martins M. Hospital mortality in older patients in the Brazilian Unified Health System, Southeast region. Rev Saúde Pública. 2018;52:69. doi: 10.11606/S1518-8787.2018052000146

9. Kovacevic P, Dragic S, Kovacevic T, Momcicevic D, Festic E, Kashyap R, et al. Impact of weekly casebased tele-education on quality of care in a limited resource medical intensive care unit. Crit Care. 2019 Jun 14;23:220. doi: 10.1186/s13054-019-2494-6

10. Vukoja M, Riviello ED, Schultz MJ. Critical care outcomes in resource-limited settings. Curr Opin Crit Care. 2018 Oct;24(5):421-7. doi: 10.1097/MCC.0000000000000528

11. Garnelo L, Lima JG, Rocha ESC, Herkrath FJ. Access and coverage of primary health care for rural and urban populations in the northern region of Brazil. Saúde Debate. 2018;42(Spec No):81-99. doi: 10.1590/0103-11042018S106

12. Associação de Medicina Intensiva Brasileira (AMIB). CENSO AMIB 2016 [Internet]. São Paulo: AMIB; 2017 [acesso em 2021 abr 26]. Disponível em: https://www.amib.org.br/fileadmin/user_upload/amib/2018/marco/19/Analise_de_Dados_UTI_Final.pdf

13. Cavalcanti AN, Pinto KDC, Maia EMC. Perfil de pacientes adultos em unidades de terapia intensiva do Nordeste brasileiro. Rev Portal Saúde Soc [Internet]. 2019 [cited 2021 Apr 26];4(2):1113-25. Available from: https://www.seer.ufal.br/index.php/nuspfamed/article/view/6455 
Perfil clínico, sociodemográfico e preditores de óbito em unidade de terapia intensiva | 18

14. Melo EM, Barbosa AA, Silva JLA, Studart RMB, Lima FET, Veras JEGLF, et al. Evolução clínica dos pacientes em uso de ventilação mecânica em unidade de terapia intensiva. Rev Enferm UFPE On Line [Internet]. 2015 [cited 2021 Apr 26];9(2):610-16. Available from: https://periodicos.ufpe.br/revistas/revistaenfermagem/article/view/10379

15. Santos FRQ, Machado MN, Lobo SMA. Adverse outcomes of delayed intensive care unit admissions from the emergency room. Rev Bras Ter Intensiva. 2020;32(1):92-8. doi: 10.5935/0103-507X.20200014

16. Junqueira RMP, Duarte EC. Fatores associados à chance para a mortalidade hospitalar no Distrito Federal. Epidemiol Serv Saúde [Internet]. 2013 [acesso em 2021 abr 26];22(1):29-39. Disponível em: http://scielo.iec.gov.br/scielo.php?script=sci_abstract\&pid=S1679-49742013000100003\&lng=pt\&nrm=is

17. Siow WT, Liew MF, Shrestha BR, Muchtar F, See KC. Managing COVID-19 in resource-limited settings: critical care considerations. Crit Care. 2020 Apr 22;24:167. doi: 10.1186/s13054-020-02890-x

18. Vargas MAO, Peter E, Luz KR, Barlem ELD, Ventura CAA, Nascimento ERP. Management of bed availability in intensive care in the context of hospitalization by court order. Rev Latinoam Enferm. 2020;28:e3271. doi: 10.1590/1518-8345.3420.3271

19. Diogo LP, Bahlis LF, Wajner A, Waldemar FS. Decreased mortality in patients hospitalized due to respiratory diseases after installation of an intensive care unit in a secondary hospital in the interior of Brazil. Rev Bras Ter Intensiva. 2015 Sept;27(3):235-9. doi: 10.5935/0103-507X.20150043

20. Goldwasser RS, Lobo MSC, Arruda EF, Angelo SA, Silva JRL, Salles AA, et al. Difficulties in access and estimates of public beds in intensive care units in the state of Rio de Janeiro. Rev Saúde Pública. 2016;50:19. doi: 10.1590/S1518-8787.2016050005997

Editora Científica: Tânia Solange Bosi de Souza Magnago

Editora associada: Alexa Pupiara Flores Coelho

\section{Autor correspondente}

Josefine Busanello

E-mail: josefinebusanello@unipampa.edu.br

Endereço: Unipampa - BR 472 - Km 585 - Caixa Postal 118 - Uruguaiana, RS

CEP: 97501-970 
19 | Busanello J, Quevedo EG, Escobal APL, Pinto DM, Silveira NP, Mocellin LP

\section{Contribuições de Autoria}

\section{1 - Josefine Busanello}

Concepção ou desenho do estudo/pesquisa; Análise e/ou interpretação dos dados; e Revisão final com participação crítica e intelectual no manuscrito.

\section{2 - Erica Gomes Quevedo}

Concepção ou desenho do estudo/pesquisa; Análise e/ou interpretação dos dados; e Revisão final com participação crítica e intelectual no manuscrito.

\section{3 - Ana Paula de Lima Escobal}

Análise e/ou interpretação dos dados; e Revisão final com participação crítica e intelectual no manuscrito.

\section{4 - Deisy Mello de Pinto}

Análise e/ou interpretação dos dados; e Revisão final com participação crítica e intelectual no manuscrito.

\section{5 - Natália Pinto Silveira}

Análise e/ou interpretação dos dados; e Revisão final com participação crítica e intelectual no manuscrito.

\section{6 - Lucas Pitrez Mocellin}

Análise e/ou interpretação dos dados; Revisão final com participação crítica e intelectual no manuscrito.

\section{Como citar este artigo}

Busanello J, Quevedo EG, Escobal APL, Pinto DM, Silveira NP, Mocellin LP. Perfil clínico, sociodemográfico e preditores de óbito em unidade de terapia intensiva. Rev. Enferm. UFSM. 2021 [Acesso em: Ano Mês Dia]; vol.11 e46: 1-19. DOI: https://doi.org/10.5902/2179769263048 